\title{
Combustion of Multi-Component Drops during Flame Spray Pyrolysis
}

\author{
Malte F. B. Stodt ${ }^{1,2}$, Jan Derk Groeneveld ${ }^{1}$, \\ Lutz Mädler $^{1,4}$, Johannes Kiefer ${ }^{2,3}$, Udo Fritsching ${ }^{1,3,4}$ \\ ${ }^{1}$ Leibniz Institute for Materials Engineering IWT, Badgasteiner Str. 3, 28359 Bremen, \\ Germany \\ ${ }^{2}$ Technische Thermodynamik, Universität Bremen, Badgasteiner Str. 1, 28359 Bremen, \\ Germany \\ ${ }^{3}$ MAPEX Center for Materials and Processes, Universität Bremen, Am Fallturm 1, 28359 \\ Bremen, Germany \\ ${ }^{4}$ Faculty of Production Engineering, Universität Bremen, Bibliothekstr. 1, 28359 Bremen, \\ Germany \\ ${ }^{*}$ Corresponding author: m.stodt@iwt.uni-bremen.de
}

\begin{abstract}
In this work, experimental investigations of the drop dynamics and in-flight droplet disruptions ( $\mu$-explosions) during flame spray pyrolysis (FSP) and corresponding single drop experiments using solvents with high volatility differentials are carried out. High-speed shadowgraphy recordings of flame sprays with precursor/solvent mixtures of iron nitrate and a solvent mixture of ethanol $\left(T_{b}=351 \mathrm{~K}\right)$ and 2-ethylhexanoic acid $\left(T_{b}=501 \mathrm{~K}\right)$ revealed the occurrence of distinct $\mu$-explosions in the spray in high frequencies. Severe instabilities leading to the formation of bubbles, bags and large ligament structures are observed. Furthermore, multiple jetting events with channels releasing vapor are visualized. It is found that the high multidirectional momentum caused by the $\mu$-explosion leads to fast drop rotations without changing the flow direction of the drops that might result in aspheric flame shapes and, thus, in presumably asymmetric heat balances of the drop. A comparison of $\mu$-explosions in the spray and single drop experiments revealed that the break up mechanisms are identical, indicating that the type of $\mu$-explosion is independent on local flow conditions. However, drops in single drop experiments require more time to reach the first $\mu$-explosion $(t \approx 3 \mathrm{~ms})$ which might be attributed to the larger initial drop size and lower gas phase temperatures compared to drops in the FSP experiments $(t \leq 250$ $\mu s)$.
\end{abstract}

\section{Keywords}

flame spray pyrolysis, microexplosions, spray combustion, droplet dynamics

\section{Introduction}

Flame spray pyrolysis (FSP) is an established technique for the gas phase synthesis of functional nanoparticles $[1,2]$. The countless materials that can be produced from a broad spectrum of possible FSP precursors available provide a large potential on the production of functional nanoparticles at laboratory and industrial scale. The liquid atomization of such precursors in a coaxial atomizer is expected to be a key process in FSP since short breakup length and time scales set the initial conditions for the flame reaction and a fast precursor release $[3,4,5]$ for the particle nucleation that take place at a few miliseconds. In order to ensure a fast precursor release, high-enthalpy solvents with boiling points $\left(T_{b}\right)$ above $373 \mathrm{~K}$ are attractive. These ensure high gas phase temperatures for the production of homogeneous powders as reported for several solvent mixtures for spray flames and single droplet experiments so far $[6,7]$. Furthermore, from a chemical point of view, most of these solvent combinations consist of a volatile alcohol compound and a high enthalpy carboxylic acid or carbonate, such as 2-ethylhexanoic acid $(E H A)$ or hexanoates $[5,8,9,10,11]$. These combinations enable the formation of metal 
carboxylates [9] that, on one hand stabilize the precursor solution, and on the other hand enable the metal ions to be released homogeneously into the vapor phase at high temperatures. The utilization of low cost alcohols, such as ethanol or 1-propanol further reduce the cost of raw materials significantly. The kinetics, as well as the local conditions (e.g. $d_{\mathrm{D}}, T_{\mathrm{D}}, T_{\mathrm{G}}, c_{\mathrm{D}, \text { precursor }}$ ), of the precursor release in single droplet experiments, as well as in spray flames are not understood, yet. While pure solvent droplets burn quasisteadily and uniformly, single droplet experiments [12] have shown that droplets containing metal precursor undergo severe droplet explosions especially when high differences in volatility between the compounds are present. Rosebrock et al. proposed three main steps for the combustion of droplets containing metal precursor [12]: i) diffusion burning of the low volatile components; ii) viscous shell formation by decomposition of metal precursor on the droplet surface; iii) droplet disruption by nucleation. These $\mu$-explosions significantly accelerate the evaporation and precursor release as numerous secondary drops are formed that undergo subsequent cascade-like explosions. It is therefore expected that $\mu$-explosions play a major role for the synthesis of homogeneous nanoparticles. However, little is known about the influence of the process conditions $\left(d_{\mathrm{D}}, u_{\mathrm{D}}, u_{\mathrm{G}}, T_{\mathrm{D}}, T_{\mathrm{G}}\right)$ on the occurence of $\mu$-explosions and about their occurence within spray flames. In our prior work [4] we observed in high-speed recordings of the iron flame luminosity bright sparks in the optical accessible spray flame boundary that are linked to large $\mu$-explosions. Detailed phase Doppler anemometry (PDA) measurements revealed the formation of bimodal droplet size distributions (DSDs) that are suspected to be the result of $\mu$-explosions. The aim of this work is to prove the unequivocal occurence of $\mu$-explosions during the FSP process by high-speed shadowgraphy imaging (HSSI).

\section{Materials and Methods}

The experiments were carried out using a $0.5 \mathrm{M}$ solution of FNT (iron(III) nitrate nonahydrate, Merck kGaA, Germany) in a mixture (35:65 vol.) of EtOH (ethanol absolute, VWR Chemicals, Radnor, USA) and EHA (2-ethylhexanoic acid, Sigma-Aldrich, USA). The chemicals were used as purchased from commercial sources. To prevent uncertainties caused by excess volumes during the solvation, the iron(III) nitrate nonahydrate was completely dissolved in the solvent and then diluted to yield solutions with a molarity of $0.5 \mathrm{M}$.

\section{Experimental Setup}

In Fig. 1 the setup of the HSSI experiment is illustrated. The setup consists of a monochromatic pulsed laser diode for back light illumination (Cavilux Smart, Cavitar: wavelength $\lambda=640$ $\mathrm{nm}$, pulse length $=10 \mathrm{~ns}$ ), that is synchronized with a high-speed camera (Vision Research Phantom VEO 710L: $94.000 \mathrm{fps}, 448 \times 112 \mathrm{Px}$ ). A telecentric long distance microscope (MML3HR110DVI-43F) is mounted on the high-speed camera to obtain a magnification of $3 \mathrm{x}$. The overall resolution power of the setup is established using a 1951 USAF resolution test target resulting in an optical resolution of $64(\mathrm{lp} / \mathrm{mm})$. Measurements are performed along the axial centerline of the spray flame from 20 to $70 \mathrm{~mm}$ height above the nozzle (HAB). At 30 and 40 $\mathrm{mm} \mathrm{HAB}$, also experiments at a radial position of $r=2 \mathrm{~mm}$ are conducted. It is noteworthy that the resolution of the contrast of drop and the background illumination is possible only for drops with $d_{\mathrm{D}}<7 \mu \mathrm{m}$. For the spray flame formation, the standardized SpraySyn burner, which is a coaxial atomizer unit equipped with a concentrical pilot flame, is utilized applying the standard operation conditions described in the work of Schneider et al. [13]. For a detailed description of the operation conditions and nozzle geometry please revise [4, 13].

The single drop combustion was conducted using a piezoelectric drop generator ejecting uniform single droplets $(\approx 112 \mu \mathrm{m})$ in a cuvette $(10 \times 10 \times 45 \mathrm{~mm})$ with a frequency of $10 \mathrm{~Hz}$. The drops were ignited by spark under standard conditions in a co-flowing oxygen atmosphere $(0.5$ $\mathrm{L} \min ^{-1}$ ). The optical setup used for high speed imaging was the same as used for the spray but using a white light source instead of the pulsed laser. 


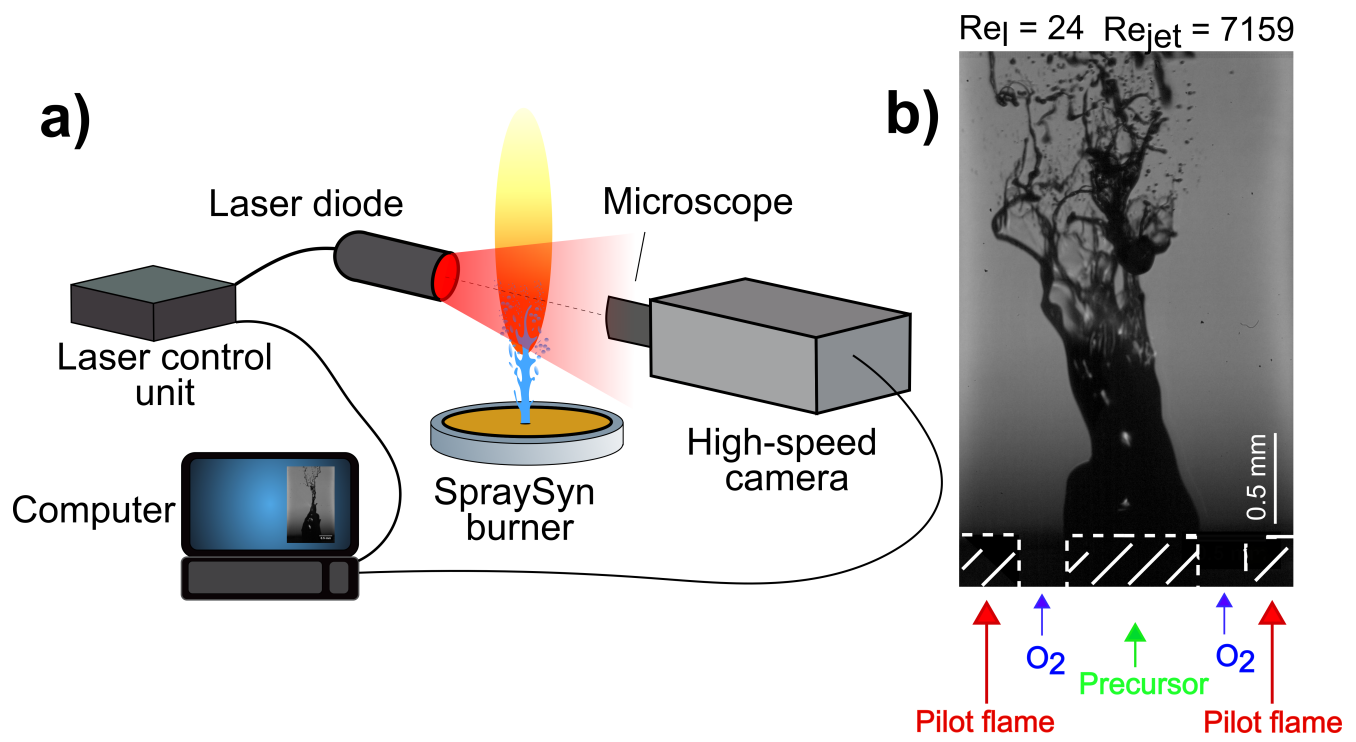

Figure 1. a) Scheme of the experimental setup for HSSI measurements in a spray flame. b) HSSI of the primary atomization of an ethanol spray flame with the liquid and jet Reynolds numbers of the present experiments.

\section{Dimensionless Quantities}

The relevant Reynolds and Weber numbers that are used to describe the flow conditions in the present spray flame are:

$$
\begin{aligned}
& \operatorname{Re}_{\mathrm{l}}=u_{1, \text { exit }} \rho_{\mathrm{D}} D_{c} \eta_{\mathrm{D}}^{-1}, \\
& \operatorname{Re}_{\mathrm{D}}=u_{\mathrm{D}} \rho_{\mathrm{G}} d_{D} \eta_{\mathrm{G}}^{-1}, \\
& \operatorname{Re}_{\text {jet }}=u_{\mathrm{G}, \text { exit }} \rho_{\mathrm{G}} 2 s \eta_{\mathrm{G}}^{-1} \\
& \overline{\mathrm{W}}_{\text {aero }}=\rho_{\mathrm{G}}\left(\bar{u}_{\mathrm{G}}-\bar{u}_{\mathrm{D}}\right)^{2} d_{\mathrm{D}, 10} \sigma^{-1},
\end{aligned}
$$

where $\mathrm{Re}_{\mathrm{l}}, \mathrm{Re}_{\mathrm{D}}, \mathrm{Re}_{\mathrm{G}}$ and $W \mathrm{We}_{\mathrm{aero}}$ are the liquid Reynolds number, the drop Reynolds number, the jet Reynolds number and the aerodynamic droplet Weber number, respectively. The quantities of $\bar{u}_{\mathrm{G}}, \bar{u}_{\mathrm{D}}$ and $d_{\mathrm{D}, 10}$ are obtained via detailed PDA measurements with the setup according to our prior work [4].

\section{Results and Discussion}

Fig. 2 shows sequences of several $\mu$-explosions visualized by HSSI along the axial centerline of the flame spray applying $2 \mathrm{~mL} \mathrm{~min}{ }^{-1}$ feed rate of $\mathrm{FNT}+\mathrm{EtOH}+\mathrm{EHA}$ and $10 \mathrm{~L} \mathrm{~min}^{-1}$ dispersion gas $\left(\mathrm{O}_{2}\right)$. The corresponding $\overline{\mathrm{W}} \mathrm{e}_{\text {aero }}$ numbers are listed in table 1 to estimate the averaged local flow conditions for the drops. The magnitude of $\bar{W} e_{\text {aero }} \approx 10^{-3}$ indicates that the aerodynamic forces acting on the drops are too low to deform drops or even lead to secondary atomization events [14, 15]. Visible drop deformations in the present experiments are therefore likely caused by spontaneous disruptions. The local differences in $\bar{W} e_{\text {aero }}$ numbers are mainly driven by changing relative velocities between drops and carrier gas [4]. 
Table 1. Local flow conditions. The mean drop velocities are adopted from Stodt et al. [4].

\begin{tabular}{|c|c|c|}
\hline $\mathrm{HAB} / \mathrm{mm}$ & $\overline{\mathrm{W}}_{\text {aero }} \times 10^{-3} /-$ & $\overline{\mathrm{u}}_{\mathrm{D}} / \mathrm{m} \mathrm{s}^{-1}$ \\
\hline 20 & 6.1 & 115.1 \\
30 & 3.5 & 111.5 \\
40 & 2.8 & 104.5 \\
50 & 6.1 & 92.7 \\
60 & 3.6 & 77.3 \\
70 & 3.5 & 62.8 \\
\hline
\end{tabular}

Directly after primary and secondary atomization of the liquid at $\mathrm{HAB}=20 \mathrm{~mm}$ high drop concentrations with spherical drops are observed, as shown in Fig. 2. This region is characterized by high drop concentrations and group combustion effects, as described by Eslamian et al. and Stodt et al. [3, 16], with ongoing flame ignition of individual drops that flow without any perturbation. Further downstream $(30 \mathrm{~mm} \leq \mathrm{HAB} \leq 70 \mathrm{~mm})$ significant surface instabilities and $\mu$-explosions are observed througout the recordings. Representative $\mu$-explosions are illustrated in Fig. 2 that are continuously observed. From these images we can deduce that most of the visible disruptions initiate with a distinctive bubble formation that results from the spontaneous nucleation of vapour of the low volatile compound inside the drop (EtOH). These bubbles continuously grow before leading to surface instabilities in form of oscillations, channels and even bag formations. These formations of vapour channels may also lead to jetting events, where the vapour release through the channel lead to the development of fine ligaments that disintegrate into secondary drops. It is noteworthy that under the present flow conditions, the diameter of such bubbles can grow to a multiple of the initial drop diameter before fragmentation. This might be attributed to a high fraction of the low volatile compound EtOH [17] as well as possible coalescence of bubbles inside the drop. These bubble breakups involve, as shown in Fig. 2 at $\mathrm{HAB}=70 \mathrm{~mm}$, bag growth, bag rupture and typically a ring breakup. Scenarios including bubble growths and subsequent surface ruptures leading to multiple jetting events are also observed (see example at $\mathrm{HAB}=60 \mathrm{~mm}$ ). Whether the bubble growth results in a bag-like breakup or in a jetting event, may presumably depend on the vapor bubble dynamics inside the droplet. A drop with the coalescence of multiple micro-bubbles inside the drop forming a large bubble may favour a bag-like breakup mechanism, while single micro-bubbles result in channeling and jetting [18]. The resulting secondary drops are expulsed by the momentum of the vapour and transported to the surroundings forming clouds of secondary drops that evaporate fast undergoing cascade-like $\mu$-explosions.

In contrast to single drop experiments, the relative high shear stress and high gas viscosity in the jet inhibits the exploding drop to change its flow direction. In fact, numerous drops (see examplary drop in Fig. 3) were observed that experienced a rotational motion caused by the radial momentum from a $\mu$-explosion. The rotation of the drops is further induced by high shear rates in the jet. Oscillations induced by the formation of a Kármán vortex street behind the drop are unlikely $\left(\operatorname{Re}_{\mathrm{D}} \ll 1000\right)$. These rotations of the drops may have an influence on the flame shape and may lead to asymmetric heat balances. The time scale of the rotation can be

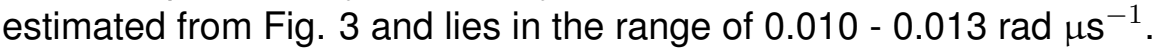



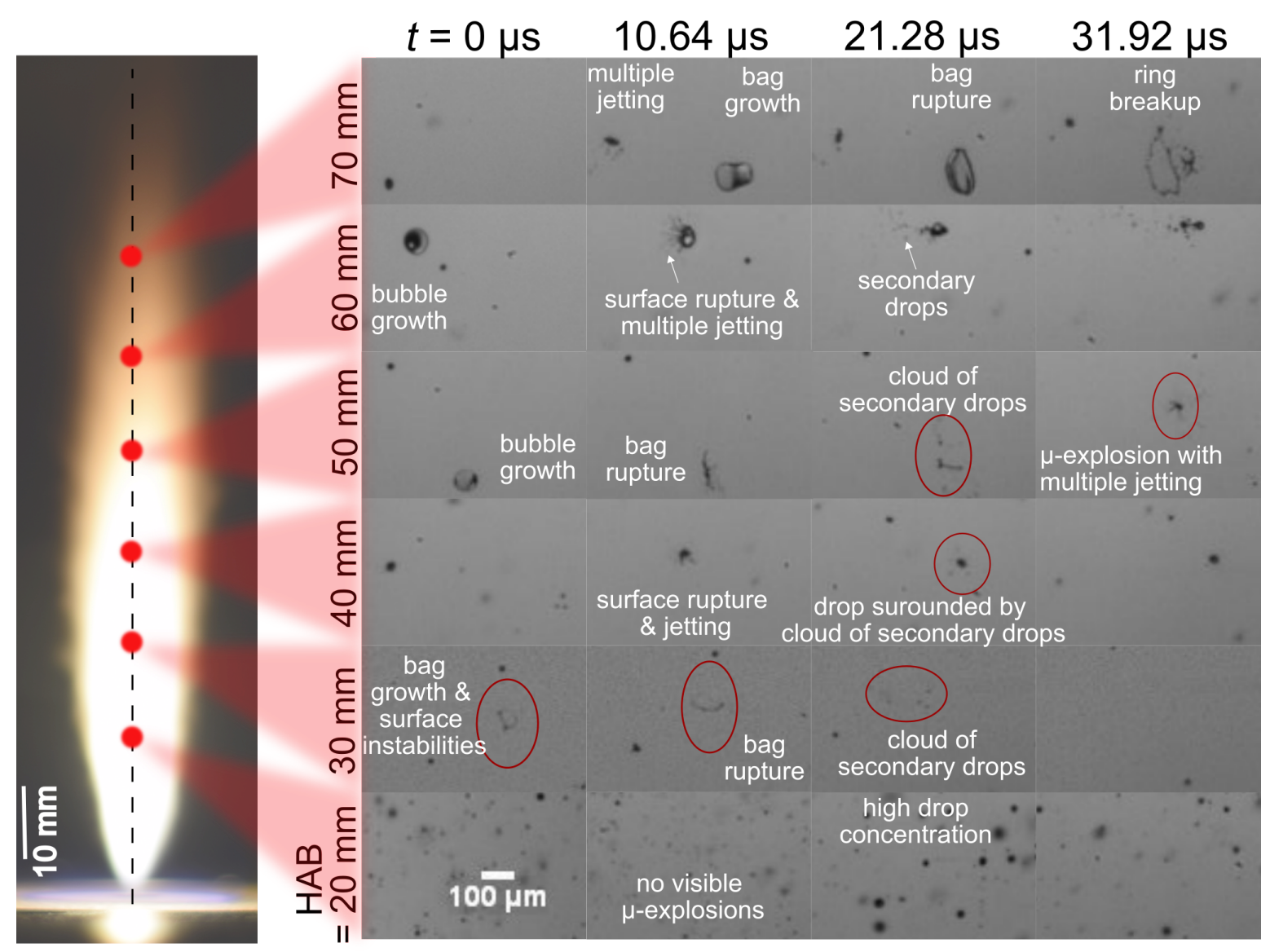

Figure 2. HSSI sequences in the flame spray at different locations on the centerline from $20 \mathrm{~mm}<\mathrm{HAB}<70 \mathrm{~mm}$. The measurement locations are indicated by red dots in the photography of the flame spray. The red circle highlight the present breakup events

\section{$\Delta t=10.64 \mu \mathrm{s}$}

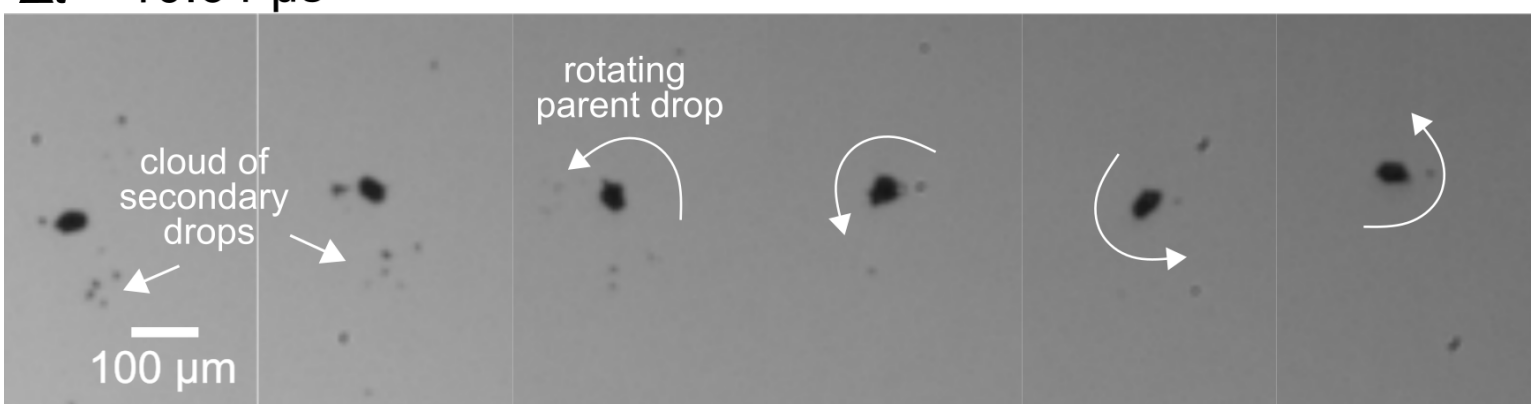

Figure 3. Rotation of an elliptic drop at $\mathrm{HAB}=40 \mathrm{~mm}$ after a partial disruption.

While in high-speed camera experiments of single drops the contrast of the flame is high enough to resolve the flame shape [12], the exposure time during the present experiments $(10 \mu \mathrm{s})$ in flame sprays was too low to capture the flame luminosity. Nevertheless, depending on the intensity of the $\mu$-explosions, frequent $\mu$-explosions of secondary drops that barely have been formed from a previous explosion are observed. This indicates that, as already observed in single drop experiments with five $\mu$-explosions in average [6], the secondary drops directly continue burning after the disruption by $\mu$-explosions with individual flames.

In order to get insights into the drop history and their time scale prior $\mu$-explosion in the present flame spray, the mean flight time $\tau_{\mathrm{F}}$ from $\mathrm{HAB}=10 \mathrm{~mm}$ up to the HAB of data point $i$ is 
estimated by

$$
\tau_{\mathrm{F}, \mathrm{i}}=\sum_{i=2}^{N} \frac{2 \Delta x_{\mathrm{i}, \mathrm{i}-1}}{\bar{u}_{\mathrm{D}, i}+\bar{u}_{\mathrm{D}, i-1}}
$$

where $\Delta x_{\mathrm{i}, \mathrm{i}-1}$ represents the local distance between the data points $i$ and $i+1$. The mean flight time $\tau_{\mathrm{F}}$ for the present spray is displayed in Fig. 4. As observed in the HSSI experiments, $\mu$-explosions are present at distances of $\mathrm{HAB} \geq 30 \mathrm{~mm}$ which is equivalent to a time scale of about $\tau_{\mathrm{F}} \geq 200 \mu \mathrm{s}$. The shaded area indicates qualitatively the time range where the $1 \mathrm{st}$ and subsequent cascade-like $\mu$-explosions occur.

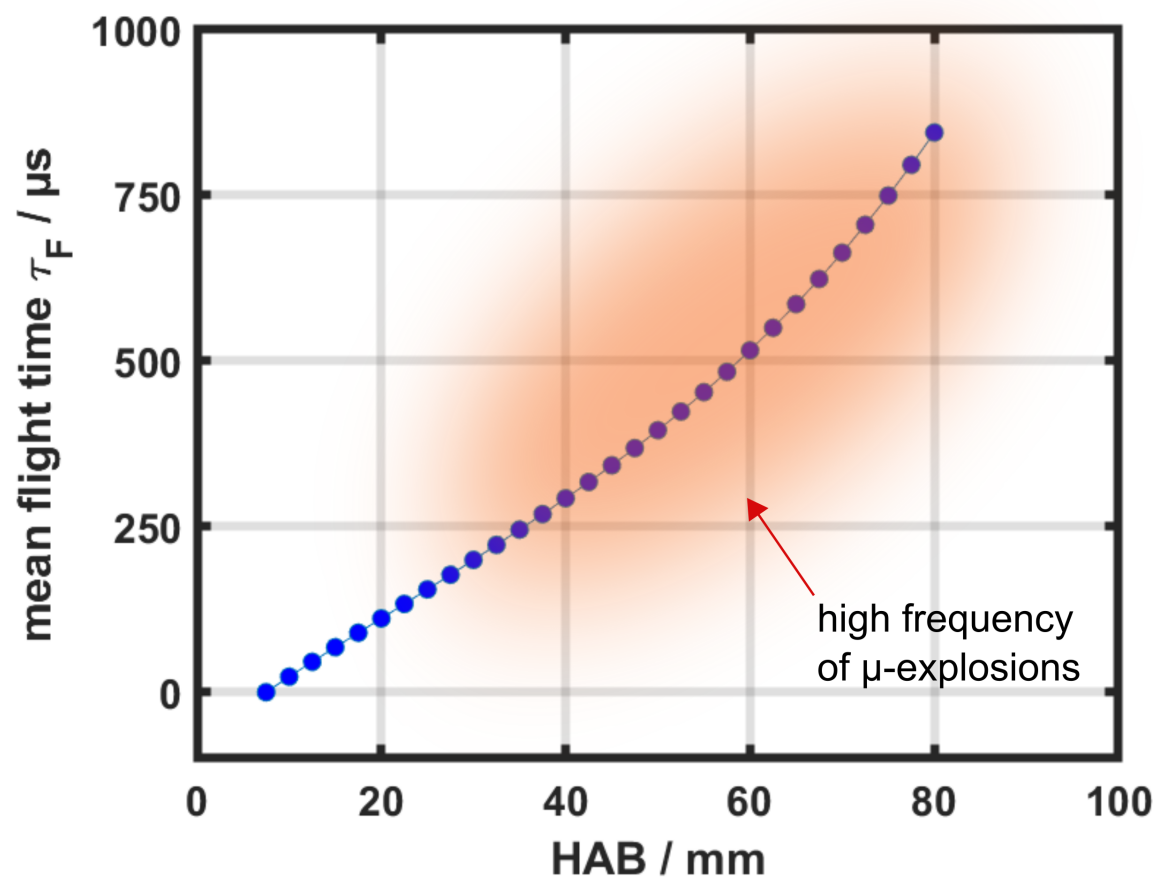

Figure 4. Mean flight time $\tau_{F}$ of the drops along the axial centerline. The shaded area indicates qualitatively the region where high frequencies of $\mu$-exlosions were observed.

In order to investigate the influence of local flow and temperature conditions, single drop experiments were performed with the same precursor and solvent solution (0.5 M FNT+EtOH+EHA) and with constant initial diameters of $d_{\mathrm{D}, 0}=112.2 \pm 0.6 \mu \mathrm{m}$. Figure 5 shows the results for the single drop experiments and reveal multiple and severe $\mu$-explosions. As can be observed in Fig. 5a the drops follow the classical $d^{2}$-law. The shaded area illustrates the normalized relative time scale at which the drops undergo the first $\mu$-explosions. The quantified time from the ignition of the drop up to the first $\mu$-explosion is displayed in Fig. 5b. The histogram reveales that the investigated $\mu$-explosions occur after a time of $t \approx 3 \mathrm{~ms}$. In comparison with similar single drop experiments in literature $[12,19]$ this is a short time scale, indicating that the present precursor solution is a appropriate system for further $\mu$-explosion experiments in sprays and single drop experiments. Fig. $5 \mathrm{c}$ shows a HSSI sequence of an examplary sample with several disintegration phenomena in one sequence. After a first catastrophic $\mu$-explosion with multipe jetting, the parent drop experiences the formation of bubbles inside. Slight perturbations are observerd which either arise from bubble dynamics inside the droplet or surface instabilities in 
form of corrugations. A second drop disruption occurs forming a ligament with surface instabilities that lead to the pinch-off of drops and a final disintegration of the ligament structure. The secondary drops are following the momentum of the explosion and are transported to the surroundings.
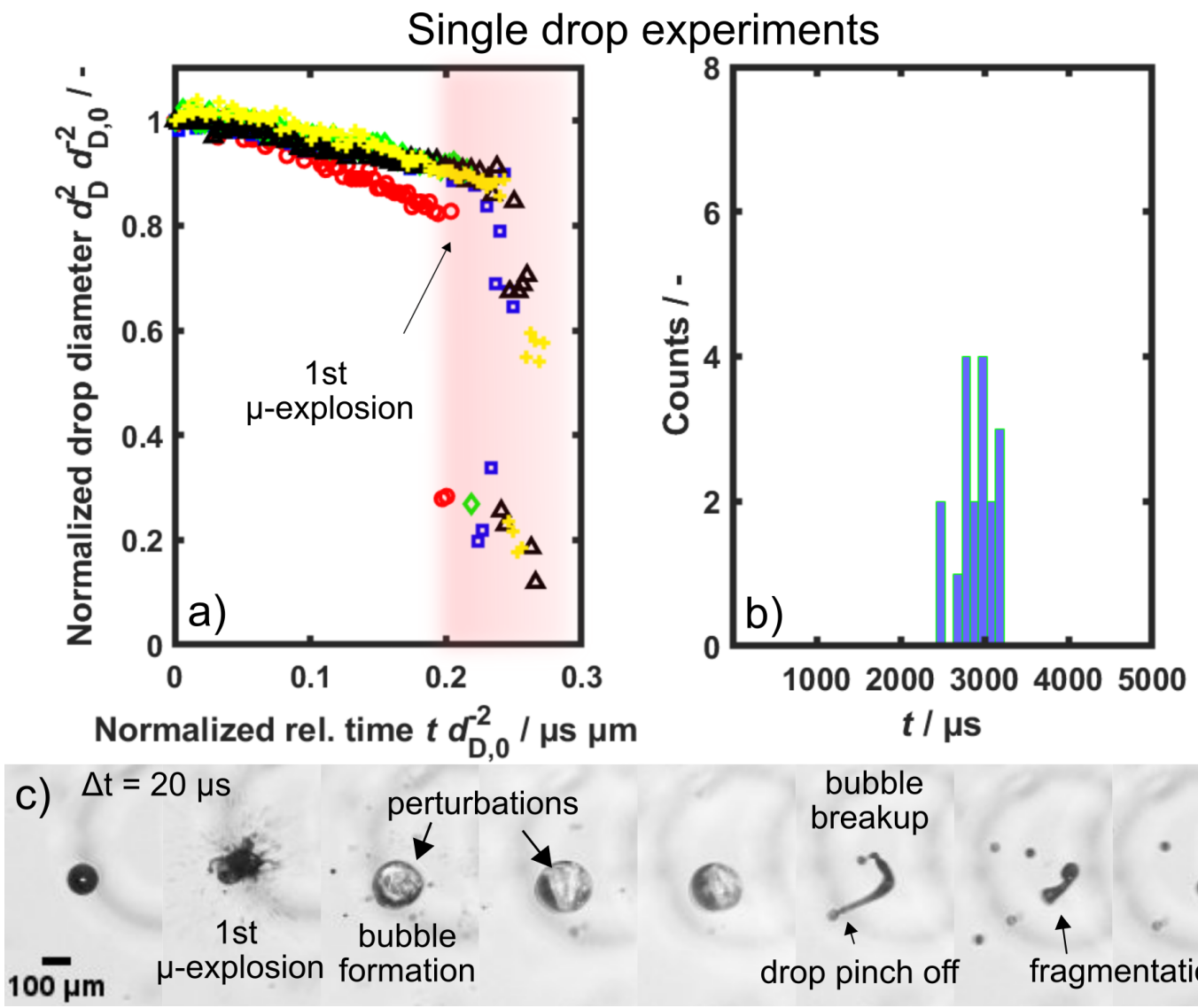

Figure 5. a) Evolution of normalized drop size for 5 samples. The shaded area illustrates the region of the 1 st $\mu$-explosion. b)
Histogram of the time of a single drop before its 1 st $\mu$-explosion involving 18 samples. c) Examplary sequence of HSSI of a
single drop during a $\mu$-explosion.

It is noteworthy that the time scales of $\mu$-explosions in the single drop experiments are significantly larger than in the present flame spray. Whether this is caused by the different flow conditions and temperature levels or by the larger initial drop sizes (slower heat \& mass transfer) remains an open question that might be resolved in future works by experiments and numerical simulations. Nevertheless, the breakup mechanisms during $\mu$-explosions are similar in both experiments.

\section{Conclusions}

The presented experimental data prove the presence of $\mu$-explosions during flame spray pyrolysis using high-speed shadowgraphy imaging. The results reveal that the outcomes and intensities of $\mu$-explosions are heterogeneous and the sizes, numbers and shapes of secondary drops vary independent on the local position in the spray. The flow direction of the parent as well as the secondary drops remains unchanged in the sprays. The multidirectional momentum of the $\mu$-explosions leads to oscillations and to distinct rotation effects with rotational velocities as high as approximately $0.01 \mathrm{rad}_{\mu \mathrm{s}^{-1}}$. The flight time of drops before their first $\mu$-explosion in the spray is estimated to be $\tau_{\mathrm{F}} \geq 250 \mu \mathrm{s}$. This time scale is significantly lower than for single drop experiments, which might be a result of large differences in gas-phase temperatures and in initial drop diameters. In order to understand the breakup mechanisms, bubble dynamics during $\mu$-explosions as well as the influence of $\mu$-explosions on the final nanoparticle properties, 
further experiments in the spray and with single drops are planned.

\section{Acknowledgements}

The financial support of this project through the German Research Foundation (DFG) within the priority program SPP 1980 SpraySyn under grants KI 1396/6-1, MA 3333/14-1 and FR 912/42-1 is gratefully acknowledged. We would also like to thank the European Research Council (ERC) under grant agreement "ReSuNiCo 786487".

\section{Nomenclature}

$\begin{array}{ll}D_{c} & \text { inner capillary diameter } \\ d_{\mathrm{D}} & \text { droplet diameter } \\ d_{\mathrm{D}, 10} & \text { droplet arithmetic mean diameter } \\ u_{\mathrm{G}, \text { exit }} & \text { gas velocity at nozzle outlet } \\ u_{1, \text { exit }} & \text { liquid velocity at capillary outlet } \\ \bar{u}_{\mathrm{D}} & \text { droplet mean velocity } \\ \bar{u}_{\mathrm{G}} & \text { gas mean velocity } \\ s & \text { width of annular gap } \\ T_{\mathrm{D}} & \text { droplet temperature } \\ T_{\mathrm{G}} & \text { gas-phase temperature } \\ c_{\mathrm{D}, \text { precursor }} & \text { precursor concentration in droplet } \\ \rho_{\mathrm{D}} & \text { droplet density } \\ \rho_{\mathrm{G}} & \text { gas density } \\ \sigma & \text { surface tension } \\ \eta_{\mathrm{G}} & \text { dynamic viscosity } \\ \tau_{\mathrm{F}} & \text { mean flight time }\end{array}$

[1] L. Mädler, H.K. Kammler, R. Mueller, and S.E. Pratsinis. J. Aerosol Sci., 33(2):369-389, 2002.

[2] L. Mädler, Wendelin J. Stark, and S.E. Pratsinis. J. Mater. Res., 18(1):115-120, 2003.

[3] M.F.B Stodt, J. Kiefer, and U. Fritsching. Exp. Fluids, 60(8):125, 2019.

[4] M.F.B. Stodt, J. Kiefer, and U. Fritsching. Atomization Sprays, 30(11), 2020.

[5] M. Bieber, R. Tischendorf, H.J. Schmid, M.A. Reddemann, and R. Kneer. Proc. 29th ILASS-Eur., 2019.

[6] H Li, C.D. Rosebrock, N. Riefler, T. Wriedt, and L. Mädler. Proc. Combust. Inst., 36(1):1011-1018, 2017.

[7] C.D. Rosebrock, T. Wriedt, L. Mädler, and K. Wegner. AIChE J., 62(2):381-391, 2016.

[8] R Tischendorf, M Simmler, C Weinberger, M Bieber, M Reddemann, F Fröde, J Lindner, H Pitsch, R Kneer, M Tiemann, et al. J. Aerosol Sci., p. 105722, 2020.

[9] M.F.B. Stodt, M. Gonchikzhapov, T. Kasper, U. Fritsching, and J. Kiefer. Phys. Chem. Chem. Phys., 21(44):24793-24801, 2019.

[10] M.F.B. Stodt, C. Liu, S. Li, L. Mädler, U. Fritsching, and J. Kiefer. Proc. Combust. Inst., doi:10.1016/j.proci.2020.06.092, 2020.

[11] S. Angel, J. Neises, M. Dreyer, K. Friedel Ortega, M. Behrens, Y. Wang, H. Arandiyan, C. Schulz, and H. Wiggers. AlChE J., 66(1):e16748, 2020.

[12] C.D. Rosebrock, N. Riefler, T. Wriedt, L. Mädler, and S.D. Tse. AIChE J., 59(12):45534566, 2013.

[13] F. Schneider, S. Suleiman, J. Menser, E. Borukhovich, I. Wlokas, A. Kempf, H. Wiggers, and C. Schulz. Rev. Scientific Instr., 90(8):085108, 2019.

[14] P.H. Clay. Proc. R. Acad. Sci. Amsterdam, volume 43, p. 852, 1940.

[15] J.O. Hinze. AlChE J., 1(3):289-295, 1955.

[16] M. Eslamian and M.C. Heine. Nanotechnol., 19(4):045712, 2008. 
[17] D.C.K. Rao, S. Karmakar, and S. Basu. Sci. Rep., 7(1):1-15, 2017.

[18] D.C.K. Rao, S. Karmakar, and S. Basu. Phys. Fluids, 30(6):067101, 2018.

[19] H. Li, C.D. Rosebrock, Y. Wu, T. Wriedt, and L. Mädler. Proc. Combust. Inst., 37(1):1203$1211,2019$. 